\title{
A NEW ONTOLOGICAL PERSPECTIVE FOR INTEGRATION OF SOCIAL AND PHYSICAL ENVIRONMENTS: DISABILITY AND REHABILITATION CONTEXT
}

\author{
Amin Gharebaghi, Mir Abolfazl Mostafavi \\ Center for research in Geomatics, Laval university, Quebec, Canada - (amin.gharebaghi.1@ulaval.ca), (mir- \\ abolfazl.mostafavi@scg.ulaval.ca)
}

Commission II, ICWG II/IV

KEYWORDS: Disability, Environment, Social and Physical dimensions, Semantics, Ontology

\begin{abstract}
:
Social dimension of environment is an important aspect that should be reflected in research works related to studying the interactions between human and the environment. However, this dimension is usually neglected when representing the environment in geographic information systems for different applications. For instance, disability as a result of the interaction between human and environment is influenced by social and physical dimensions of environment. Although, this aspect is highlighted in most conceptual disability models by defining various taxonomies of the environment, from ontological perspective justifying and connecting social dimension to the physical dimension of the environment is not clearly determined. Integrating social dimension of the environment with its physical dimension for disability studies is a challenging task, which is the main objective of the present study. Here, we review some of the disability models and their perspective about classifying the environment. Then, from ontological perspective, their limitations are discussed and a new approach for the classification of concepts form the environment is presented. This approach facilitates and simplifies integration of social dimension in ontologies for more effective assessment of disability issue in Geographic Information System.
\end{abstract}

\section{INTRODUCTION}

Disability has been defined and treated differently in the periods before and after 1970s. Before 1970s, disability was treated as a result of or related to diseases and injuries. This approach was called medical model of disability. Criticisms of this definition has led to the development of a new approach for disability in late 1970s (Edwards et al., 2014). This vision is called social model of disability, where impairment is used for physical condition of body and the disability is defined in relation with the society as a significant factor in causing disability. For instance, society can force isolation and exclusion of an individual from social participation. As a consequence, in social models, socio-economic systems are the main focus while medical models consider body's system functions to study the disability (Edwards et al., 2014).

The environment is considered as one of the most central elements in the disability models. For instance, the Disability Creation Process (DCP) model (Fougeyrollas, 1998), the International Classification of Functioning, Disability and Health (ICF) model (World Health Organization, 2001), and the Institute of Medicine (IOM) model (Brandt Jr and Pope, 1997) as the well-known disability models include the environment as the main element. All of them are in agreement that the environment plays a fundamental role in disability creation process and hence "disability cannot be fully understood without considering the environmental context" (Whiteneck et al., 2004). However, these models slightly vary in details of their taxonomies that describe and specify environmental factors. The prominent similarity in all these taxonomies is the inclusion of physical and social factors. Although they categorize the environment into social and physical environments and identify many concepts in relation to disability process, they do not fully conceptualize their relations. For example, in the DCP model, the interactions between environmental and human factors of people with disability is presented in general terms and relations among specific factors have not been explicitly determined.

Ontologies are defined as specification of conceptualization. A conceptualization means an abstract and simplified model that includes all pertinent elements. These elements will differ with respect to the domains and applications. For instance, in the studies related to the interaction between human and environment such as in disability studies physical and social aspects of environment should be integrated. Significant research has been made to model the interaction between the environment and human factors. (Warren, 1984), (Jonietz et al., 2013), (Matthews et al., 2003), (Kasemsuppakorn and Karimi, 2009) are some examples. Several ontologies are also defined to conceptualize the environment in different domains. For instance, Berdier (2011), Timpf (2002), and Sen (2008) developed ontologies for mobility context. A serious weakness in most of mentioned studies, however, is that they rarely take into account the social aspect of the environments and they mostly focused on the physical characteristics of the environment.

Social factors include political, economic, social and cultural systems. The social factors are subdivided into "politicaleconomic" and "sociocultural" elements in the some of disability models such as in DCP model. The political-economic factors include the structures and operational modes and services of different governing systems, whereas the sociocultural factors indicate the structures and operational modes of an individual's relationships with other members of society. Norms, policies, and financial issues are some examples of social aspects. Associating these parameters to physical environment in disability creation process is a 
challenging issue, which is important that provides a better foundation for the assessment of disability in tools such as geographic information systems (GIS). To address this issue, classifying the environment into physical and social dimensions by disability models is argued and drawbacks of different classifications for defining ontologies are analyzed. Then, in order to embed the social environment in ontologies and construct a more exhaustive semantic network, a different perspective is presented. This approach facilitates and simplifies the environment conceptualization for disability studies.

The remainder of the paper is organized as follows. Section two reviews three well-known disability models and explains the conceptualization of the environment using ontologies in these models. Section three explains the social-physical perspective to conceptualize the environment. Section four proposes a new perspective to link social environment to physical environment from an ontological perspective and section five presents conclusions and future work.

\section{BACKGROUND}

As mentioned previously, in late 1970s, social model of disability was developed. In this model, impairment is used for physical condition of body and the disability is defined in relation with society. This model highlights the significant role of environment in the life of people with disabilities. The environment, in physical rehabilitation and disability models, is subdivided into "social and biological/functional arenas, perhaps in parallel with the social and medical models" (Edwards 2014). This vision, however, implicates challenging issues in terms of developing ontologies for conceptualization of environment and integrated consideration of social and physical factors in GIS for disability studies.

\subsection{Disability models taxonomies}

Several disability models highlighted the role of environment in social participation of persons with disabilities. The first model that clearly articulates the role of environment is the DCP model that has been introduced by Fougeyrollas et al. (1998). These authors argue that the social participation of persons with disabilities is the result of interactions between personal and environmental factors. In this model, the environmental factors have been divided into social and physical factors that can be either obstacles or facilitators. Figure 1 presents different interactions between these factors.

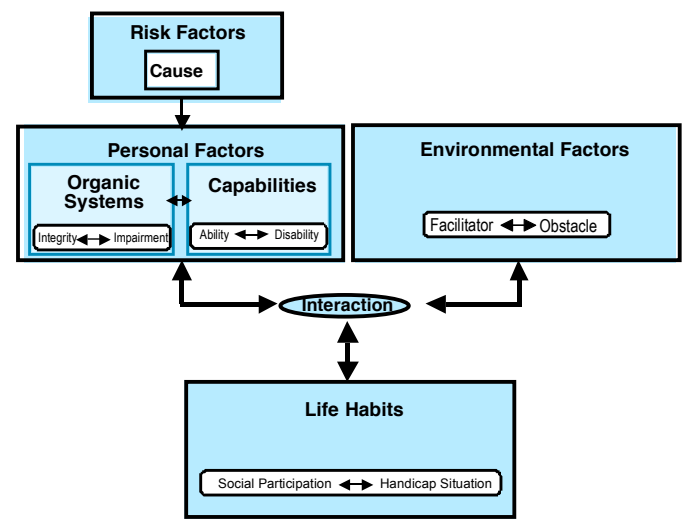

Figure 1. Disability Creation Process Model (Fougeyrollas et al. (1998))
Similar to DCP, the IOM introduced by Brandt Jr \& Pope (1997) presents the disability as a result of interactions between personal and environmental factors. This model also classifies the environment into two subclasses of "physical" and "social and psychological" environment. Brandt Jr \& Pope (1997) conceptualized the social environment to include political, economical, and cultural factors. They specified the psychological environment as intrapersonal environment.

World health organization as an important international group presented ICF model that is consistent with two previously mentioned models in the main parts including environmental and personal factors (Figure 2). In this model, the environmental factors have been divided into "physical, social, and attitudinal environments in which people live and conduct their lives" (Whiteneck et al., 2004).

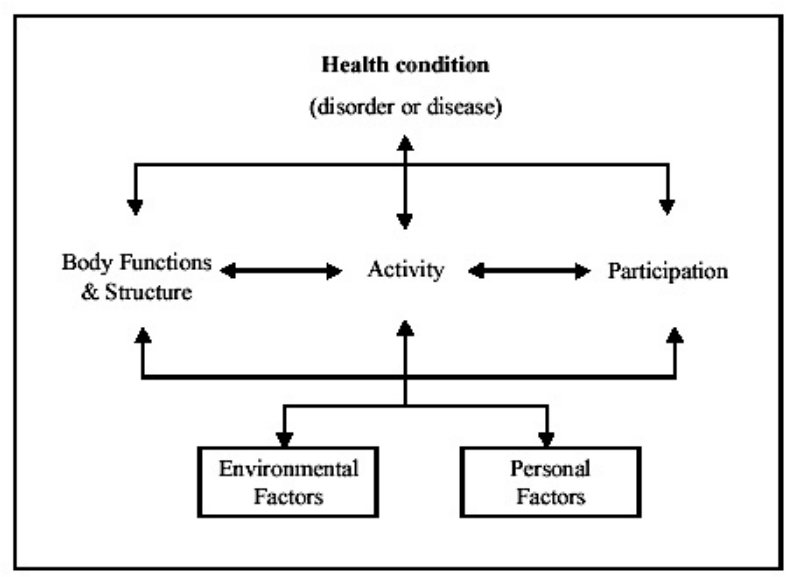

Figure 2. International Classification of Functioning (World Health Organization, 2001)

Although the mentioned models present diverse taxonomies for the environmental factors by defining different categories, all taxonomies include physical, political, economical, social, and cultural factors. The DCP model classifies physical factors into natural and development sub-classes and social factors into political-economic and sociocultural sub-classes. The IOM model categorized the environment into physical and socialpsychological environment, where natural and built environments are grouped to the physical environment and cultural, psychological, political, and economic environments are counted as the elements of social-psychological environment. Finally, the ICF model also subdivided the social and physical environment into five environmental chapters of products and technology; natural environment and human-made changes; support and relationships; attitudes; and services, systems, and policies. The prominent similarity in all these taxonomies is the inclusion of physical and social factors in disability models. Furthermore, in all mentioned models in order to explain the person and environment interaction, three scales of environment described by Bronfenbrenner (1992) are assumed. These levels include micro ${ }^{1}$ (personal), meso ${ }^{2}$ (community/services), and $\operatorname{macro}^{3}$ (societal/systems)

\footnotetext{
${ }^{1}$ Micro is all environments, which can be adapted for specific individuals such as home or an office.

${ }^{2}$ Meso is the collective context, which can only be collectively designed. Meso environment is related to public places and community life.

${ }^{3}$ Macro environment is considered as societal space.
} 
environment. These taxonomies are mostly defined as global ontologies without defining specifically the relations between social and physical environments in more operational perspectives that will be discussed in the next section.

\subsection{Absence of social factors: Examples form ontologies devolved for navigation task}

Several ontologies are defined to conceptualize the environment in different domains. In the mobility context, significant works have been carried out to define navigation ontologies in an urban area. Timpf (2002) developed ontology of wayfinding with multiple transportation modes in a given path in two different perspectives of a traveler and public transportation system. Berdier \& Roussey (2007) developed three urban ontologies including, road system, urban mobility, and urban renewal ontology that contain many terms of road system, the mobility and trip, and urban renewal concepts of environment, respectively. Berdier (2011) developed an ontology related to mobility in an urban area. This ontology attempts to integrate fuzzy concepts and to connect two ontologies of road system and urban mobility. Sen (2008) presented a case study of extracting knowledge about affordances of road network entities. This case study includes notions of nested and sequential affordances. He conducted analysis of two traffic code texts based on a word co-occurrence model and discussed the possible options to integrate such information in geospatial ontologies. One of the limitations of these projects is that, most of them do not take into account the social factors - they only consider the physical characteristics of the environment and the navigation concepts themselves.

Many definitions are proposed for ontology from different perspectives. Common perspectives include the philosophical, artificial intelligence and linguistic points of view. In this paper we use the definition of ontology from artificial intelligence point of view, which indicates that ontology is "a formal, explicit specification of a shared conceptualization" (Gruber, 1993). A conceptualization means an abstract and simplified model of how people think about things in the world. A conceptual model presents the concepts, objects, and relations among them. According to (Uschold and King, 1995) and (López, 1999) the first step of conceptualization to build an ontology is "ontology capture" which means: 1) Identification of the key concepts and relationships in the domain of interest, that is, scoping. 2) Production of precise unambiguous text definitions for such concepts and relationships. 3) Identification of terms to refer to such concepts and relationships, and 4) Agreeing on all of the above". In this paper, we are going to employ this definition of ontology to impose social aspect of environment in the ontologies from computer science perspective. Indeed, employing ontological perspective will assist to associate the social concepts to physical environment. Hence, in following sections, after arguing the traditional approach of the environment classification, a new approach is presented to integrate the social concepts in ontologies.

\section{SOCIAL-PHYSICAL PERSPECTIVE}

In order to conceptualize the environment in a given domain (e.g. mobility), identifying concepts and their relationships are required. Considering social-physical division of the environment that employed in DCP, IOM, and ICF, leads to categorize concepts based on physical and social environments. Then we need to define appropriate relationships among them. In reality, there are many direct and indirect relations between entities in social and physical environments. Therefore, the relationships among the concepts should be identified one by one from social and physical environments. In addition, relationships among the concepts are not «one-to-one» or «oneto-many» and they are mostly «many-to-many» relationships (Figure 3). For example, driving culture as a social concept affects the noise, air pollution, and traffic congestion. On the other hand, traffic as a physical entity is affected by oil cost, working hours of public and private organizations as well as driving culture. Identifying all one by one relationships among the concepts from two categories and defining a many-to-many relationship among the concepts related to social and physical environments might be too complex.

It is important to note that the relation between social entities are often materialized through the physical entities. This adds more complexity in defining relationships between those concepts. For example, the culture of driving and the policy of snow removal are two social concepts that affect the movement of traffic on the streets. These concepts have connections with streets, cars, snow, air pollution, noise and some other concepts. By employing the social-physical perspective, the culture of driving or the policy of snow removal are classified into the social category and street, cars, snow, air pollution, and noise are classified into the physical category, separately. Then, we need to define relationships among these concepts which is not only a challenging but also time-consuming process. To avoid these complexities, instead of social-physical perspective, we propose to employ a new standpoint that we call "naturedevelopment " perspective.



Figure 3. Social-physical perspective of environment

\section{NATURE-DEVELOPMENT PERSPECTIVE}

In nature-development perspective, each concept belongs either to natural environment (such as forest, tree, and snow) or to developed environment (such as sidewalk, building, and transportation means). We have considered these two categories because the relation between social factors with natural elements (ex. tree) is different from those of man-made elements (ex. building). Environmental concepts, either nature or development, have two dimensions including physical and social dimensions where the social dimension affects the physical aspect. Indeed, the social dimension is made to manage human's everyday live (Edwards et al., 2014). The physical dimension is related to the physical properties whereas the social dimension is related to sociocultural and politicaleconomical aspects. In this approach, each concept has physical and social properties where these properties are intertwined and are related to each other (Figure 4). For instance, the policy of weekly trash collecting from the sidewalks in Quebec City is a 
social rule that can be considered as a social property of trash/sidewalk, which affects the accessibility of the sidewalk. This policy was mentioned as a social concept in social-physical division in DCP that is used as a social property here.

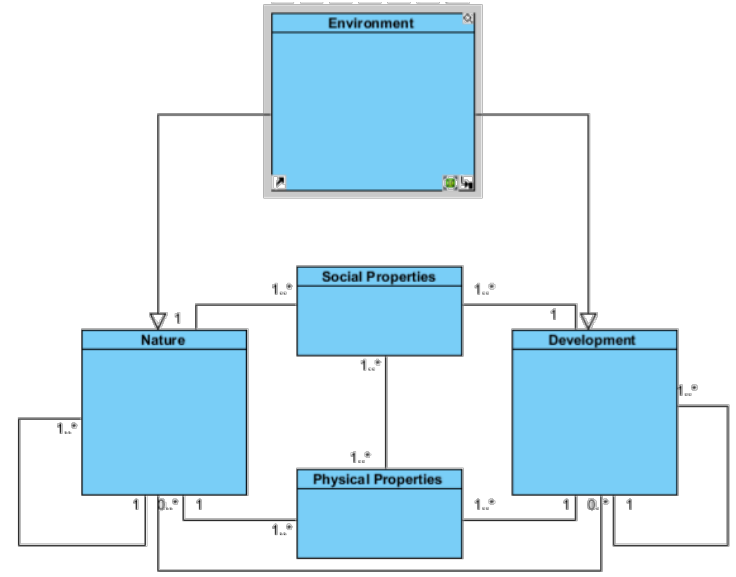

Figure 4. Nature-Development perspective

In nature-development approach, after identifying a concept in natural or developed categories, the physical and social properties related to the concept in a given domain (e.g. mobility) should be determined. Then, relationships between the properties are defined. Furthermore, different concepts (natural or built concepts) have influence on each other. In the next step, relationships among the concepts should be defined. Thus, the nature-development approach includes three steps: 1) determination of the concepts in each category 2) defining relationships among concepts (natural/developed concepts) and 3 ) defining the social and physical properties of a concept and their relationship. Hence, instead of employing the socialphysical division, if the nature-development approach is applied, cascading down the ontology that embraces the social and physical properties will be significantly simplified.

Each concept in built or natural environment has relationships with other concepts of the environment. Relationships among the concepts affect the social and physical properties of them. For instance, relationships of a sidewalk with a city and with a restaurant patio are analyzed. A pedestrian network as a built concept "is placed in" a city and a restaurant patio as a dynamic furniture "is located on" the sidewalk. Locating the patio on the sidewalk along the side of a street in a city leads to have different properties in compare to sidewalk in the other city. They can be different in shape, dimension, and color norms, opening hours, municipality policies, etc. For instance, in some cities, the regulation of municipality allows to occupy the major part of sidewalks by restaurants. Diversity of these properties can affect the results of interaction between human and environment; in this case, the accessibility of the sidewalks for persons with disabilities. Indeed, these regulations can produce the obstacles for wheelchair users and passing the sidewalk becomes more difficult (Figure 5).

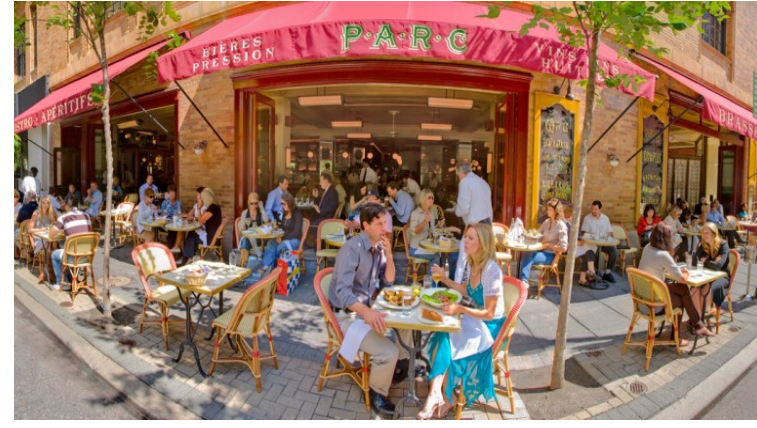

Figure 5. Sidewalk occupation by a restaurant patio ${ }^{1}$

Therefore, for analyzing the accessibility of pedestrian networks for persons with disability, not only the physical and social properties of the pedestrian networks, but also the influence of other concepts in relationship with them should be considered, too. Figure 6 shows the "pedestrian network" in relation with other concepts. Among the concepts, relationship with "restaurant patio" is highlighted. Therefore, in the process of concepts, relationships among concepts are very important and should be considered.

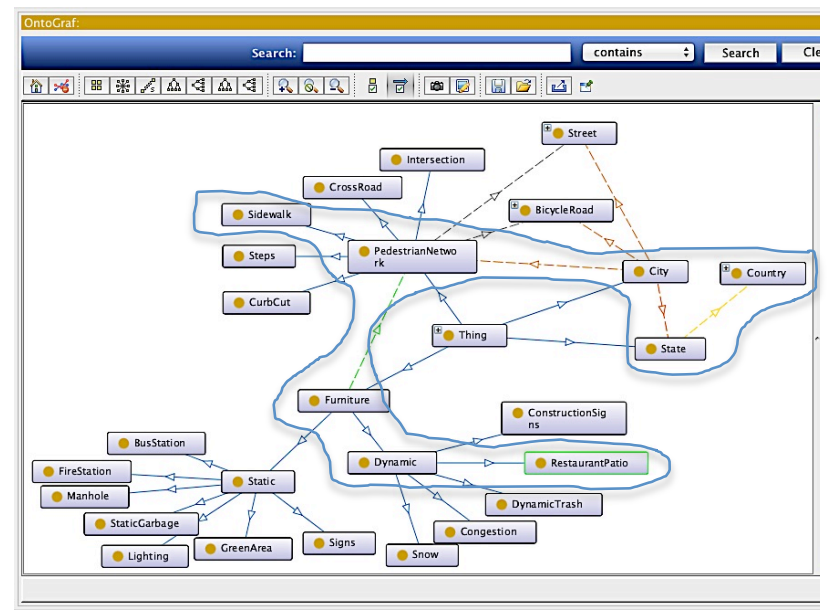

Figure 6. "Pedestrian network" in relation with "Restaurant patio"

To better understand the notion of nature-development categorization, an example is explained as follows. The "sign" concept belongs to the built environment category. Signs are made to provide information for users. By nature-development perspective, the concept of "sign" has two dimensions that include physical and social dimensions. Signs in physical perspective, have a material such as Aluminum, Acrylic, and Magnetic. They have dimensions, colors and many other physical properties. In addition, many policies, norms, maintenance issues, and financial aspects have been defined for them to be properly used by public. These properties constitute social properties of sign concept. For instance, the shape, color, dimension, height, placement, and font size of sidewalk signs are different than street signs that have been designed for pedestrian and drivers, respectively. Figure 7 shows the

\footnotetext{
${ }^{1} \mathrm{http}: / /$ www.visitphilly.com/articles/philadelphia/alfrescodining/
} 
pertinent social and physical properties of "sign" concept. As explained, by employing the nature-development perspective we can simply find the social concepts that are related to a given concept and use them as social properties of that concept. In the following sub-section, we will explain the impact of social dimension on the obstacle/facilitator using «influence scale» for concepts.

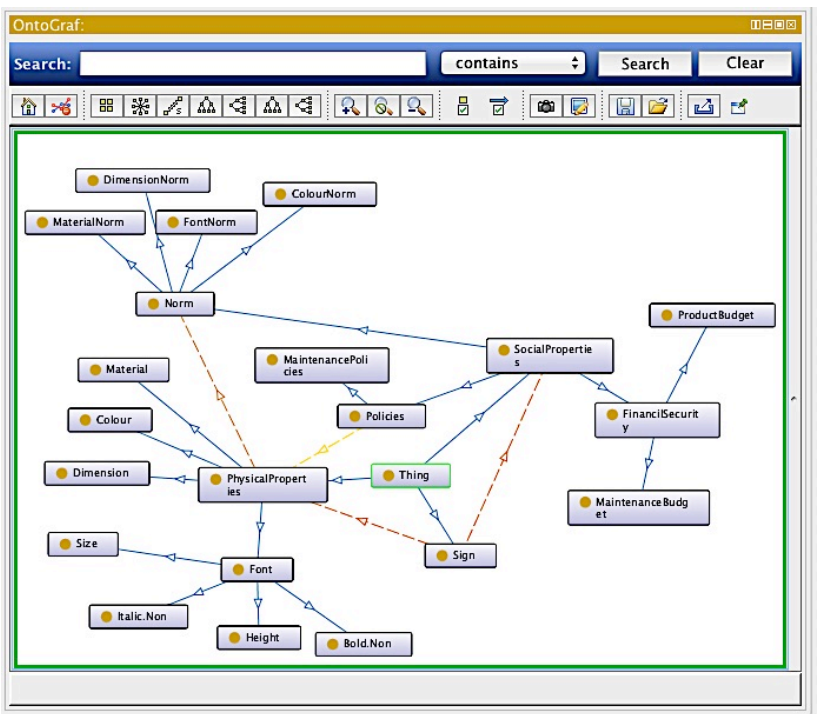

Figure 7. Semantic network of "Sign" (including social and physical properties)

\subsection{Obstacles or facilitators: the impact of social dimension}

Some environmental concepts can be qualified either as obstacle $^{1}$ or facilitator ${ }^{2}$ with different influence scale. Figure 8 shows a qualitative scale for obstacles and facilitators to Measure the Quality of the Environment (MQE) that has been proposed by Fougeyrollas (2002). The MQE scale "aims at evaluating the environment's influence on the accomplishment of a person's daily activities in relation to his/her abilities and limits"(Fougeyrollas, 2002). The MQE estimates the influence scale of environment from "major obstacle" to "major facilitator".

Although user capabilities are one of the effective elements in determining the level of obstacles and facilitators, imposing social properties on the concepts can affect the level of obstacle or facilitator role of an object in different contexts. Employing the nature-development approach provides a unique framework that includes social and physical properties of a concept and allows us to analyze the effects of social properties on a concept influence scale. For instance, snow is inherently considered as an obstacle for the mobility of persons with motor disability. Imposing policies to remove snow from the pedestrian network would affect the influence scale of this obstacle.

\footnotetext{
${ }^{1}$ An obstacle refers to "an environmental factor that hinders the accomplishment of life habits when interacting with personal factors" (Fougeyrollas et al., 1998).

${ }^{2}$ A facilitator refers to "an environmental factor that contributes to the accomplishment of life habits when interacting with personal factors" (Fougeyrollas et al., 1998).
}



Figure 8 . The influence scale: the impact of social and physical properties on qualification of obstacles and facilitators

(Fougeyrollas, 2002)

\subsection{Multi-scale perspective}

As previously mentioned, the interactions of people with their environment occurs in different micro, meso and macro scales (Bronfenbrenner, 1992). Employing the nature-development perspective also allows identifying relationships among concepts at different levels. For example, the public transportation as a developed environment can be discussed in two scales. Buses, trains, subways, and taxies as transportation means make the physical dimension of transportation and are discussed in meso level. The physical dimension of transportation includes many physical properties such as dimension, capacity, and material. Transportation system that makes the social dimension of transportation is connected to macro environment. Transportation system includes concepts such as working hours, speed limitations, stop positions, and passengers number limitation.

Figure 9 visualizes the cascade down of "transportation". As it is shown, there are some relations between physical properties (in meso level) and social properties (in macro level). For instance, the limitation of passenger numbers from macro level is related to the dimension of vehicle.

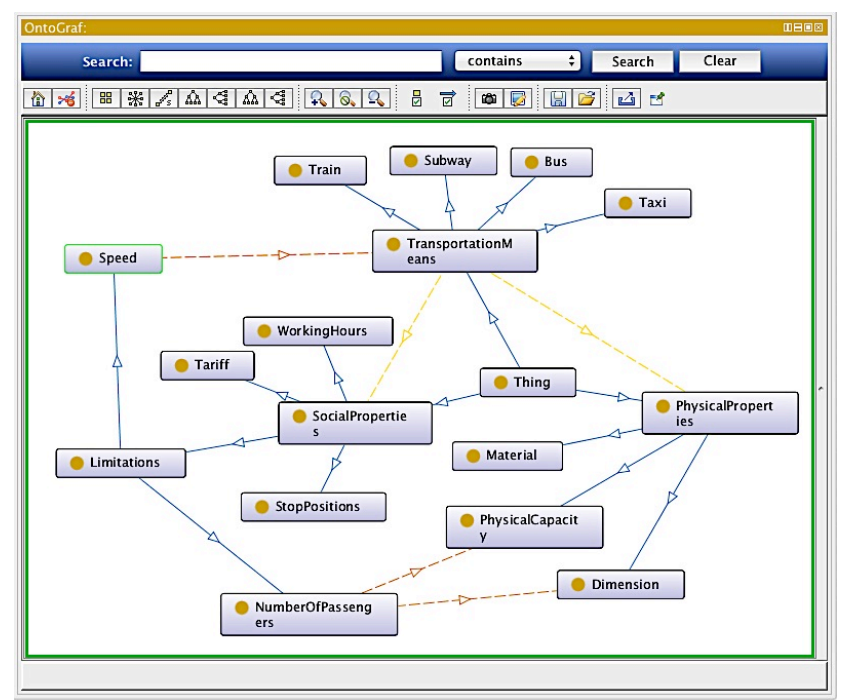

Figure 9. Transportation in different levels

With the perspective presented in this paper, it will be more feasible to consider not only the influence of social dimension of the environment in the assessment of disability but also we are capable to model it in a multidimensional context. This method will help more concretely qualify and quantify the interactions between human factors with environmental factors composed of both social and physical parameters. 


\section{CONCLUSION AND FUTURE WORK}

Integrating social concepts as an important component of the environment in disability ontologies was studied in this paper. Three well-known disability models were reviewed and the social dimension of environment and its influence on the assessment of disability was highlighted. Then, the traditional classification of environment into social-physical categories was argued. This paper showed that employing the socialphysical division of the environment complicates the process of developing ontologies, especially in terms of defining relationships between social and physical entities. This is fundamental for modeling and the interaction between human and its social and physical environments in GIS. To address this problem, a new approach called "nature-development" division was presented. Using this approach allows us to use the physical and social dimensions of the concepts in the same framework. In addition, using this perspective allows us to analyze the effects of social properties on the obstacle/facilitator influence scale. Further research will be carried out to develop specific mobility ontology for persons with disabilities based on the results of current study.

\section{ACKNOWLEDGEMENT}

This research was supported by MobiliSIG project thanks to the grant provided by CHIR and NSERC of CANADA. We are thankful to all the members of MobliSIG project team for their valuable contribution to the discussions that helped us in this paper.

\section{REFERENCES}

Berdier, C., 2011. An Ontology for Urban Mobility, in: Ontologies in Urban Development Projects. Springer, pp. 189196.

Berdier, C., Roussey, C., 2007. Urban ontologies: The towntology prototype towards case studies, in: Ontologies for Urban Development. Springer, pp. 143-155.

Brandt Jr, E.N., Pope, A.M., 1997. Enabling America:: Assessing the Role of Rehabilitation Science and Engineering. National Academies Press.

Bronfenbrenner, U., 1992. Ecological systems theory. Jessica Kingsley Publishers.

Edwards, G., Boucher, N., Fougeyrollas, P., Grenier, Y., MacFadyen, B., Morales, E., Noreau, L., Vincent, C., 2014. Disability, rehabilitation research and post-cartesian embodied ontologies - has the research paradigm changed, in: Research in Social Science and Disability, Special Issue on Addressing the Environmental Context of Disability. doi:10.1108/S1479354720140000008005

Fougeyrollas, P., 1998. The Quebec classification: disability creation process. INDCP, International network on the disability creation process; CSICIDH.

Fougeyrollas, P., 2002. Measure of the Quality of the Environment, Version 2.0. INDCP.

Fougeyrollas, P., Bergeron, H., Cloutier, R., Côté, J., St Michel,
G., 1998. The Québéc Classification: Disability Creation Process, Recherche et formation.

Gruber, T., 1993. A Translation Approach to Portable Ontology Specifications. Knowl. Acquis.

Jonietz, D., Schuster, W., Timpf, S., 2013. Modelling the Suitability of Urban Networks for Pedestrians: An AffordanceBased Framework, in: Geographic Information Science at the Heart of Europe. Springer, pp. 369-382.

Kasemsuppakorn, P., Karimi, H. a., 2009. Personalised routing for wheelchair navigation, Journal of Location Based Services. doi:10.1080/17489720902837936

López, F., 1999. Overview Of Methodologies For Building Ontologies. Proc. IJCAI99 Work. Ontol. Probl. Methods Lessons Learn. Futur. Trends CEUR Publ. 1999, 1-13. doi:10.1.1.39.6002

Matthews, H., Beale, L., Picton, P., Briggs, D., 2003. Modelling Access with GIS in Urban Systems (MAGUS): capturing the experiences of wheelchair users. Area 35, 34-45. doi:10.1111/1475-4762.00108

Sen, S., 2008. Use of affordances in geospatial ontologies, in: Towards Affordance-Based Robot Control. Springer, pp. 122139.

Timpf, S., 2002. Ontologies of Wayfinding: a Traveler' s Perspective 9-33.

Uschold, M., King, M., 1995. Towards a Methodology for Building Ontologies. Methodology. doi:10.1.1.55.5357

Warren, W.H., 1984. Perceiving affordances: visual guidance of stair climbing. J. Exp. Psychol. Hum. Percept. Perform. 10, 683-703.

Whiteneck, G.G., Harrison-Felix, C.L., Mellick, D.C., Brooks, C. a, Charlifue, S.B., Gerhart, K. a, 2004. Quantifying environmental factors: a measure of physical, attitudinal, service, productivity, and policy barriers. Arch. Phys. Med. Rehabil. 85, 1324-35. doi:10.1016/j.apmr.2003.09.027

World Health Organization. (2001) International classification of functioning, disability and health: ICF. 